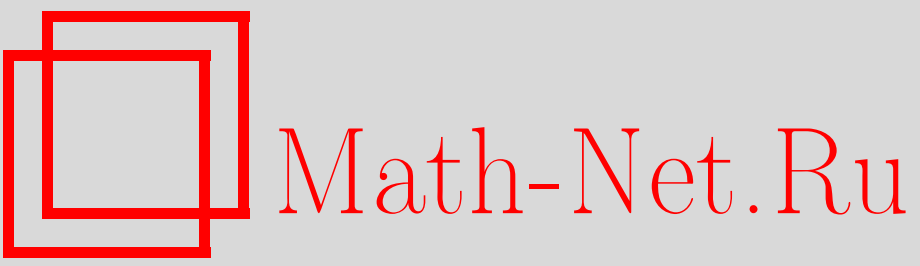

А. А. Славнов, К. В. Степаньянц, Универсальная инвариантная перенормировка для суперсимметричных теорий, ТМФ, 2003, том 135, номер 2, 265-279

DOI: https://doi.org/10.4213/tmf189

Использование Общероссийского математического портала Math-Net.Ru подразумевает, что вы прочитали и согласны с пользовательским соглашением

http://www . mathnet.ru/rus/agreement

Параметры загрузки:

IP: 54.196 .121 .252

26 апреля 2023 г., 17:06:56 
ТЕОРЕТИЧЕСКАЯ

И МАТЕМАТИЧЕСКАЯ

ФИЗИКА

Том 135, № 2

май, 2003

(C) 2003 г.

А. А. Славнов* , К.В. Степаньянц ${ }^{\dagger}$

\section{УНИВЕРСАЛЬНАЯ ИНВАРИАНТНАЯ ПЕРЕНОРМИРОВКА ДЛЯ СУПЕРСИММЕТРИЧНЫХ ТЕОРИЙ}

\footnotetext{
Предлагается универсальная калибровочно-инвариантная схема перенормировки для суперсимметричных теорий. Эта схема применяется для суперсимметричной квантовой электродинамики.
}

Ключевые слова: регуляризация, суперсимметрия, перенормировка, контрчлены.

\section{1. ВВЕДЕНИЕ}

В данной работе исследуется проблема построения явно калибровочно-инвариантной процедуры перенормировки для суперсимметричных калибровочных теорий.

Специфической особенностью суперсимметричных калибровочных теорий является неполиномиальная форма действия. Единственная возможность записать действие в полиномиальной форме состоит в использовании калибровки Весса-Зумино. Однако калибровка Весса-Зумино не является суперсимметричной и не позволяет использовать суперграфы для вычислений квантовых поправок.

В суперсимметричных калибровках сушествует бесконечное число примитивно расходяшихся диаграмм, что формально делает теорию неперенормируемой. Однако можно показать, что суперсимметрия и калибровочная инвариантность сокрашают число независимых контрчленов, так что оказывается достаточным проведение перенормировки заряда и волновой функции. Соответствуюшая процедура впервые была предложена для суперсимметричной квантовой электродинамики одним из авторов [1], а затем применена к абелевым и неабелевым моделям в работах [2]-[4]. Однако доказательство, приведенное в этих работах, основано на предположении о возможности использования промежуточной регуляризации, сохраняюшей симметрии теории. Тем не менее наиболее популярные калибровочно-инвариантные регуляризации, такие как размерная регуляризация [5], размерная редукция [6] или решеточные регуляризации, нару-

* Математический институт им. В. А. Стеклова РАН, Московский государственный университет, Москва, Россия. E-mail: slavnov@mi.ras.ru

${ }^{\dagger}$ Московский государственный университет, Москва, Россия. E-mail: stepan@theor.phys.msu.su 
шают суперсимметрию [7]. В принципе в суперсимметричных теориях можно использовать регуляризацию при помоши высших ковариантных производных [8]-[10], однако соответствуюшие вычисления являются достаточно сложными (см. [11], [12]).

Возможным альтернативным подходом является алгебраическая перенормировка. В рамках этого метода можно использовать произвольную регуляризацию или схему вычитаний, нарушаюшую симметрию теории. (В качестве примера можно привести обрезание петлевого импульса или дифференциальную перенормировку [13], [14].) Симметрия восстанавливается на втором шаге подбором конечных контрчленов таким образом, чтобы обеспечить выполнение обобщенных тождеств Уорда для перенормированных функций Грина. Этот метод был успешно применен к суперсимметричным калибровочным теориям в работах [15]-[17], где инвариантная перенормируемость $N=1$ и $N=2$ неабелевых суперсимметричных калибровочных моделей была доказана в рамках алгебраической перенормировки. Однако практическое использование алгебраической перенормировки достаточно сложно технически, поскольку соответствующая процедура требует подбора значительного числа (неинвариантных) контрчленов.

Недавно был предложен новый метод проведения калибровочно-инвариантной перенормировки [18], [19], который автоматически обеспечивает симметрию перенормированных функций Грина для произвольной промежуточной регуляризации. Этот метод сформулирован в виде специальной процедуры вычитаний, гарантирующей выполнение обобшенных тождеств Уорда для перенормированных функций Грина. Решая соответствуюшие обобшенные тождества Уорда, проблему перенормировки при использовании произвольной (в том числе неинвариантной) промежуточной регуляризации можно свести к явно калибровочно-инвариантной процедуре. Поэтому вместо двухшаговой алгебраической перенормировки получается одношаговый алгоритм, обеспечиваюший требуемую симметрию перенормированной теории.

В данной работе исследуется возможность обобщения этого метода для суперсимметричных калибровочных теорий. При этом детально описана перенормировка суперсимметричной квантовой электродинамики. Соответствующая процедура для неабелевых суперсимметричных калибровочных теорий в настоящий момент находится в стадии построения. Статья построена следующим образом. В разделе 2 вводятся обозначения и напоминаются некоторые сведения о суперсимметричной квантовой электродинамике. Универсальная калибровочно-инвариантная схема перенормировки построена в разделе 3 . Ее применение иллюстрируется на однопетлевом уровне в разделе 4 . Полученные результаты кратко обсуждаются в разделе 5 .

\section{2. СУПЕРСИММЕТРИЧНАЯ КВАНТОВАЯ ЭЛЕКТРОДИНАМИКА}

В суперпространстве $N=1$ суперсимметричная электродинамика описывается следуюшим действием:

$$
\begin{aligned}
S_{0}= & \frac{1}{4 e^{2}} \operatorname{Re} \int d^{4} x d^{2} \theta W_{a} C^{a b} W_{b}+\frac{1}{4} \int d^{4} x d^{4} \theta\left(\phi^{*} e^{2 V} \phi+\tilde{\phi}^{*} e^{-2 V} \tilde{\phi}\right)+ \\
& +\frac{1}{2} \int d^{4} x d^{2} \theta m \tilde{\phi} \phi+\frac{1}{2} \int d^{4} x d^{2} \bar{\theta} m \tilde{\phi}^{*} \phi^{*},
\end{aligned}
$$


где $\phi$ и $\tilde{\phi}$ - киральные суперполя, $V$ - вешественное суперполе. Суперполе $W_{a}$ в абелевом случае определяется как

$$
W_{a}=\frac{1}{16} \bar{D}\left(1-\gamma_{5}\right) D\left[\left(1+\gamma_{5}\right) D_{a} V\right]
$$

где

$$
D=\frac{\partial}{\partial \theta}-i \gamma^{\mu} \theta \partial_{\mu}
$$

- обычная суперсимметричная ковариантная производная. При этом интегрирование по суперпространству в формуле (1) определяется следуюшим образом:

$$
\begin{gathered}
\int d^{2} \theta=\frac{1}{4} \frac{\partial}{\partial \theta}\left(1+\gamma_{5}\right) \frac{\partial}{\partial \bar{\theta}}, \quad \int d^{2} \bar{\theta}=\frac{1}{4} \frac{\partial}{\partial \theta}\left(1-\gamma_{5}\right) \frac{\partial}{\partial \bar{\theta}}, \\
\int d^{4} \theta=\int d^{2} \theta d^{2} \bar{\theta}=\frac{1}{8}\left(\frac{\partial}{\partial \theta} \frac{\partial}{\partial \bar{\theta}}\right)^{2} .
\end{gathered}
$$

С точностью до полных производных эти выражения могут быть переписаны в явно суперсимметричной форме:

$$
\begin{gathered}
\int d^{4} x d^{2} \theta=-\frac{1}{4} \int d^{4} x \bar{D}\left(1+\gamma_{5}\right) D=-\frac{1}{2} \int d^{4} x D^{2}, \\
\int d^{4} x d^{2} \bar{\theta}=-\frac{1}{4} \int d^{4} x \bar{D}\left(1-\gamma_{5}\right) D=-\frac{1}{2} \int d^{4} x \bar{D}^{2} \\
\int d^{4} x d^{4} \theta=\frac{1}{4} \int d^{4} x \bar{D}^{2} D^{2}=\frac{1}{4} \int d^{4} x D^{2} \bar{D}^{2} .
\end{gathered}
$$

При этом были использованы следуюшие обозначения:

$$
D^{2} \equiv \frac{1}{2} \bar{D}\left(1+\gamma_{5}\right) D, \quad \bar{D}^{2} \equiv \frac{1}{2} \bar{D}\left(1-\gamma_{5}\right) D .
$$

Действие (1) инвариантно относительно калибровочных преобразований

$$
\begin{gathered}
V \rightarrow V-\frac{1}{2}\left(\Lambda+\Lambda^{*}\right), \\
\phi \rightarrow e^{\Lambda} \phi, \quad \phi^{*} \rightarrow \phi^{*} e^{\Lambda^{*}}, \\
\tilde{\phi} \rightarrow e^{-\Lambda} \tilde{\phi}, \quad \tilde{\phi}^{*} \rightarrow \tilde{\phi}^{*} e^{-\Lambda^{*}},
\end{gathered}
$$

где $\Lambda$ - произвольное киральное суперполе. Эта инвариантность позволяет положить ряд компонент $V(x, \theta)$ равными нулю, в результате чего данное суперполе оказывается равным

$$
V(x, \theta)=\frac{1}{2} \bar{\theta} \gamma^{\mu} \gamma_{5} \theta A_{\mu}(x)+i \sqrt{2}(\bar{\theta} \theta)\left(\bar{\theta} \gamma_{5} \chi(x)\right)+\frac{1}{4}(\bar{\theta} \theta)^{2} D(x),
$$


где $A_{\mu}$ - калибровочное поле, $\chi$ - майорановский спинор, а $D$ - вещественное скалярное вспомогательное поле. В такой калибровке, которая называется калибровкой Весса-Зумино, остаточные калибровочные преобразования зависят от единственного вещественного параметра, а действие является полиномиальным. Однако выбор калибровки Весса-Зумино явно нарушает суперсимметрию теории.

Квантование модели (1) детально описано в книге [20] и здесь не рассматривается. Необходимо только отметить, что фиксация калибровки осушествлялась при помощи добавления слагаемого

$$
S_{\mathrm{gf}}=\frac{1}{32 e^{2} \xi} \int d^{4} x d^{4} \theta D^{2} V \bar{D}^{2} V
$$

в котором $\xi$ является некоторой постоянной.

\section{3. УНИВЕРСАЛЬНАЯ КАЛИБРОВОЧНО-ИНВАРИАНТНАЯ СХЕМА ПЕРЕНОРМИРОВКИ}

В этом разделе будет построена процедура перенормировки, которая может применяться при использовании произвольной, не обязательно калибровочно-инвариантной регуляризации и приводит к перенормированным функциям Грина, которые автоматически удовлетворяют суперсимметричным обобщенным тождествам Уорда. На языке контрчленов это означает, что после такой перенормировки не нужно добавлять калибровочно-неинвариантные контрчлены и перенормировочный произвол ограничивается выбором калибровочно-инвариантных локальных членов, форма которых не фиксируется обобшенными тож дествами Уорда. При этом мы предполагаем, что регуляризация, используемая для вычислений, является явно суперсимметричной и допускает использование техники суперграфов. Предлагаемая далее схема вычитаний может быть использована для произвольных суперсимметричных моделей, сформулированных в терминах соответствуюших суперполей. Однако в данной работе детально рассмотрена только перенормировка суперсимметричной КЭД и все выписанные уравнения относятся именно к этой модели. При этом мы следуем подходу, предложенному в работах [18], [19], где была построена схема выгитаний, обеспечиваюшая автоматическое выполнение обобщенных тождеств Уорда для несуперсимметричных моделей.

Для того чтобы избежать появления инфракрасных расходимостей, мы будем работать в “диагональной” калибровке, соответствующей значению $\xi=-1$ в формуле (9). Рассмотрение произвольной калибровки требует введения дополнительной инфракрасной регуляризации, которая в абелевом случае может быть осуществлена путем добавления массового слагаемого для калибровочного поля. После вычисления интегралов необходимо положить $m_{A}=0$ (см. [21]), где $m_{A}$ - параметр инфракрасной регуляризации.

Хорошо известно [20], что индекс расходимости диаграммы, содержащей $E_{\phi}$ внешних линий киральных и антикиральных суперполей и $E_{V}$ внешних линий калибровочного суперполя, равен

$$
\omega=2-P-E_{\phi},
$$


где $P$ - число пропагаторов $\phi \phi, \tilde{\phi} \tilde{\phi}, \phi^{+} \phi^{+}$и $\tilde{\phi}^{+} \tilde{\phi}^{+}$. Поэтому расходимости присутствуют только в следующих функциях Грина ${ }^{1)}$ :

$$
\begin{gathered}
(2 \pi)^{4} \delta^{4}\left(k_{\mu}+q_{\mu}+\left(p_{1}\right)_{\mu}+\cdots+\left(p_{n}\right)_{\mu}\right) \times \\
\quad \times \Gamma\left[\left(\theta_{x_{1}}, p_{1}\right), \ldots,\left(\theta_{x_{n}}, p_{n}\right) ;\left(\theta_{y}, q\right),\left(\theta_{z},-q-p_{1}-\cdots-p_{n}\right)\right] \equiv \\
\left.\equiv \int d^{4} x_{1} \ldots d^{4} x_{n} d^{4} y d^{4} z \frac{\delta^{n+2} \Gamma}{\delta V_{x_{1}} \ldots \delta V_{x_{n}} \delta \phi_{y} \delta \phi_{z}^{+}}\right|_{V, \phi, \tilde{\phi}=0} \times \\
\quad \times \exp \left(i\left(p_{1}\right)_{\mu} x_{1}^{\mu}+\cdots+i\left(p_{n}\right)_{\mu} x_{n}^{\mu}+i q_{\mu} y^{\mu}+i k_{\mu} z^{\mu}\right) . \\
(2 \pi)^{4} \delta^{4}\left(\left(p_{1}\right)_{\mu}+\cdots+\left(p_{n}\right)_{\mu}\right) \Pi\left[\left(\theta_{x_{1}}, p_{1}\right), \ldots,\left(\theta_{x_{n}},-p_{1}-\cdots-p_{n-1}\right)\right] \equiv \\
\left.\equiv \int d^{4} x_{1} \ldots d^{4} x_{n} \frac{\delta^{n} \Gamma}{\delta V_{x_{1}} \ldots \delta V_{x_{n}}}\right|_{\phi, \tilde{\phi}, V=0} \exp \left(i\left(p_{1}\right)_{\mu} x_{1}^{\mu}+\cdots+i\left(p_{n}\right)_{\mu} x_{n}^{\mu}\right),
\end{gathered}
$$

а также в функциях $\widetilde{\Gamma}$, которые строятся аналогично функциям $\Gamma$, но дифференцирование выполняется по полям $\tilde{\phi}$. Заметим, что функции П для нечетного $n$ равны нулю, поскольку вклады диаграмм с петлями поля $\phi$ сокрашаются с соответствуюшими диаграммами, содержашими петли поля $\tilde{\phi}$.

Функции Г, Г и П удовлетворяют тождествам Уорда [4], которые в наших обозначениях могут быть записаны в следующем виде:

$$
\begin{aligned}
\left(D_{x_{1}}^{2}+\right. & \left.\bar{D}_{x_{1}}^{2}\right) \Gamma\left[\left(\theta_{x_{1}}, p_{1}\right),\left(\theta_{x_{2}}, p_{2}\right), \ldots,\left(\theta_{x_{n}}, p_{n}\right) ;\left(\theta_{y}, q\right),\left(\theta_{z},-q-p_{1}-\cdots-p_{n}\right)\right]= \\
= & 2 \bar{D}_{x_{1}}^{2} \delta^{4}\left(\theta_{y}-\theta_{x_{1}}\right) \times \\
& \times \Gamma\left[\left(\theta_{x_{2}}, p_{2}\right), \ldots,\left(\theta_{x_{n}}, p_{n}\right) ;\left(\theta_{x_{1}}, q+p_{1}\right),\left(\theta_{z},-q-p_{1}-\cdots-p_{n}\right)\right]+ \\
& +2 D_{x_{1}}^{2} \delta^{4}\left(\theta_{x_{1}}-\theta_{z}\right) \times \\
& \times \Gamma\left[\left(\theta_{x_{2}}, p_{2}\right), \ldots,\left(\theta_{x_{n}}, p_{n}\right) ;\left(\theta_{y}, q\right),\left(\theta_{x_{1}},-q-p_{2}-\cdots-p_{n}\right)\right] \\
\left(D_{x}^{2}+\right. & \left.\bar{D}_{x}^{2}\right)\left(\Pi\left[\left(\theta_{x}, p\right),\left(\theta_{y},-p\right)\right]-\frac{1}{2 e^{2} \xi} p^{2} \delta^{4}\left(\theta_{x}-\theta_{y}\right)\right)=0 \\
\left(D_{x_{1}}^{2}+\right. & \left.\bar{D}_{x_{1}}^{2}\right) \Pi\left[\left(\theta_{x_{1}}, p_{1}\right), \ldots,\left(\theta_{x_{n-1}}, p_{n-1}\right),\left(\theta_{x_{n}},-p_{1}-\cdots-p_{n-1}\right)\right]=0, \quad n>2 .
\end{aligned}
$$

При этом суперсимметричные ковариантные производные в импульсном представлении записываются как

$$
D_{x}=\frac{\partial}{\partial \bar{\theta}}-\gamma^{\mu} p_{\mu}
$$

где $p$ - импульс, соответствуюший координате $x$. Тождества Уорда для функций $\widetilde{\Gamma}$ имеют ту же структуру.

\footnotetext{
1) Заметим, что в случае использования инвариантной регуляризации расходимости в функциях П отсутствуют при $n>2$. Однако в общем случае нельзя игнорировать их существование.
} 


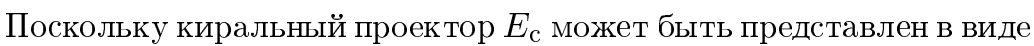

$$
E_{\mathrm{c}} \equiv \frac{1}{16 \partial^{2}}\left(\bar{D}^{2} D^{2}+D^{2} \bar{D}^{2}\right)=\frac{1}{16 \partial^{2}}\left(\bar{D}^{2}+D^{2}\right)^{2},
$$

то эти тождества связывают функции Грина с по крайней мере одной "киральной" компонентой калибровочного поля с корреляторами, имеюшими меньшее число киральных компонент калибровочного поля.

Уравнения (13)-(15) записаны для случая суперсимметричной квантовой электродинамики. Однако они имеют такую же структуру, как и тождества Уорда в неабелевых теориях. Отличие заключается в форме правой части, которая в неабелевом случае включает корреляторы с внешними линиями духов Фаддеева-Попова.

Наша стратегия может быть сформулирована следуюшим образом. Сначала рассматриваются однопетлевые диаграммы и произвольным образом перенормируются диаграммы, соответствуюшие функциям Грина в правой части уравнений (13)-(15). Затем, принимая во внимание, что в теории, не содержашей квантовых аномалий, обобшенные тождества Уорда для корреляторов, вычисляемых в произвольной регуляризации, могут нарушаться только локальными членами, определяем перенормированные функции Грина в левой части формул (13)-(15) таким образом, чтобы эти тождества выполнялись автоматически. Заметим, что такая перенормировка все же является неполной, поскольку очевидно, что обобщенные тождества Уорда допускают добавление к вершинной функции в левой части произвольный калибровочно-инвариантный контрчлен. Такие контрчлены, как обычно, являются свободными параметрами, которые могут быть зафиксированы условиями нормировки.

В случае суперсимметричной квантовой электродинамики эта процедура выглядит следующим образом. Вначале необходимо провести перенормировку однопетлевой двухточечной функции Грина суперполей материи. Члены, пропорциональные $\phi^{*} \phi$ и не содержашие $V$ в эффективном действии, имеют следуюшую структуру:

$$
4 \int d^{4} x d^{4} \theta \phi^{*}(x) \Sigma\left(\sqrt{-\partial^{2}}\right) \phi(x)
$$

Поэтому двухточечная функция Грина суперполей материи может быть записана в виде

$$
\Gamma[(x, q),(y,-q)]=\bar{D}_{x}^{2} D_{x}^{2} \delta^{4}\left(\theta_{x}-\theta_{y}\right) \Sigma(q) .
$$

Соответствуюшая перенормированная функция определяется вычитанием

$$
\Sigma^{r}(q)=\Sigma(q)-\Sigma\left(\mu_{\sigma}\right)
$$

где $\mu_{\sigma}$ - точка нормировки. 
После перенормировки двухточечной функции необходимо построить перенормированные вершинные функции Г и $\widetilde{\Gamma}$. В силу суперсимметрии функция Г может быть представлена как

$$
\Gamma[\theta, p]=\sum_{i} B_{i}(\theta, p) F_{i}(p),
$$

где через $p$ и $\theta$ обозначен весь набор аргументов этой функции, $B_{i}(\theta, p)-$ некоторые полиномы по $p$ и $\theta$, которые являются линейно независимыми комбинациями ковариантных производных, действуюшими на произведения $\delta^{4}\left(\theta_{k}-\theta_{l}\right)$, и $F_{i}(p)$ - некоторые скалярные функции внешних импульсов. Перенормировка производится при помощи вычитания из функций $F_{i}(p)$ полиномов $P_{i}(p)$. Мы будем выбирать эти полиномы таким образом, чтобы получаемые перенормированные функции Грина удовлетворяли обобщенным тождествам Уорда (13)-(15), в которых правая часть содержит перенормированные функции с меньшим числом внешних линий.

Определим “частично перенормированную” функцию

$$
\gamma^{r}[\theta, p]=\sum_{i} B_{i}(\theta, p)\left(F_{i}(p)-P_{i}(p)\right)
$$

где функции $P_{i}(p)$ являются некоторыми полиномами по внешним импульсам. Тогда левая часть уравнения (13) может быть переписана в виде

$$
\left(D_{x_{1}}^{2}+\bar{D}_{x_{1}}^{2}\right) \gamma^{r}[\theta, p]=\sum_{i}\left(D_{x_{1}}^{2}+\bar{D}_{x_{1}}^{2}\right) B_{i}(\theta, p)\left(F_{i}(p)-P_{i}(p)\right)
$$

Величины $\left(D_{x_{1}}^{2}+\bar{D}_{x_{1}}^{2}\right) B_{i}(\theta, p)$ в общем случае не являются линейно независимыми. Поэтому удобно ввести линейно независимые полиномы $Q_{j}$, пропорциональные киральным частям $B_{i}$ :

$$
\left(D_{x_{1}}^{2}+\bar{D}_{x_{1}}^{2}\right) B_{i}(\theta, p)=\sum_{j} c_{i j} Q_{j}(\theta, p)
$$

Раскладывая правую часть уравнения (13) по базису $Q_{j}$, можно записать тождество Уорда в виде

$$
\sum_{i j} c_{i j} Q_{j}(\theta, p)\left(F_{i}(p)-P_{i}(p)\right)=\sum_{j} Q_{j}(\theta, p) R_{j}(p) .
$$

Поскольку величины $Q_{j}$ линейно независимы, это уравнение эквивалентно следуюшей системе линейных уравнений:

$$
\sum_{i} c_{i j}\left(F_{i}(p)-P_{i}(p)\right)=R_{j}(p)
$$

которая представляет собой тождество Уорда (13), разложенное по линейно независимым полиномам $Q_{i}(p, \theta)$. Если полиномы $P_{i}(p)$ удовлетворяют системе $(26)$, функция 
$\gamma^{r}$, определяемая формулой (22), удовлетворяет суперсимметричному тождеству Уорда. Действительно,

$$
\left(D_{x_{1}}^{2}+\bar{D}_{x_{1}}^{2}\right) \gamma^{r}[\theta, p]=\sum_{i} c_{i j} Q_{j}(\theta, p)\left(F_{i}(p)-P_{i}(p)\right)=\sum_{j} Q_{j}(\theta, p) R_{j}(p)
$$

Заметим, что выбор $P_{i}$ не является единственным, поскольку любое решение тождества Уорда определяется с точностью до слагаемого $\Pi_{1 / 2} f$, которое из тождества Уорда не определяется. Однако, этот произвол не сушествен для нашей процедуры.

Для устранения остаточных ультрафиолетовых расходимостей достаточно вычесть из $\gamma^{r}$ калибровочно-инвариантные контрчлены $P_{g i}$ :

$$
\Gamma^{r}[p, \theta]=\gamma^{r}[p, \theta]-P_{g i},
$$

которые не могут быть зафиксированыпри помощи обобщенных тождеств Уорда. Таким образом, удается свести процедуру вычитаний при использовании произвольной регуляризации к вычитанию калибровочно-инвариантных контрчленов.

После построения функции $\Gamma_{3}^{r}$ необходимо подставить ее в правую часть перенормированного тождества Уорда (13) для функции $\Gamma_{4}^{r}$. После этого процесс повторяется. Таким образом, построена рекуррентная процедура, которая позволяет определить все функции $\Gamma_{n}^{r}$. Эта процедура иллюстрируется в следуюшем разделе на примере однопетлевой перенормировки функции $\Gamma_{3}$ при использовании регуляризации обрезанием петлевого импульса. Функции $\widetilde{\Gamma}^{r}$ строятся аналогичным образом.

На следуюшем этапе необходимо перенормировать функции Грина П, которые соответствуют диаграммам без внешних линий суперполей материи. Благодаря суперсимметрии они могут быть записаны в форме, аналогичной (21):

$$
\Pi[\theta, p]=\sum_{i} B_{i}(\theta, p) F_{i}(p)
$$

Например, функция П при $n=2$ может быть представлена в виде

$$
\Pi\left[\left(\theta_{x}, p\right),\left(\theta_{y},-p\right)\right]=F_{1}(p) p^{2} \Pi_{1 / 2} \delta^{4}\left(\theta_{x}-\theta_{y}\right)+F_{2}(p) \delta^{4}\left(\theta_{x}-\theta_{y}\right),
$$

где

$$
\Pi_{1 / 2}=-\frac{1}{16 \partial^{2}} D^{a} \bar{D}^{2} C_{a b} D^{b}
$$

В этом случае

$$
B_{1}(\theta, p)=p^{2} \Pi_{1 / 2} \delta^{4}\left(\theta_{x}-\theta_{y}\right), \quad B_{2}(\theta, p)=\delta^{4}\left(\theta_{x}-\theta_{y}\right) .
$$


Как и ранее, перенормированные функции Грина получаются при вычитании из $F_{i}(p)$ некоторых полиномов, которые выбираются таким образом, чтобы обеспечить вьполнение обобшенных тождеств Уорда для функции

$$
\Pi^{r}[\theta, p]=\sum_{i} B_{i}(\theta, p)\left(F_{i}(p)-P_{i}(p)\right)
$$

Для двухточечной функции Грина подстановка (30) в уравнение (14) дает равенство

$$
\left(F_{2}(p)-P_{2}(p)-\frac{1}{2 e^{2} \xi} p^{2}\right)\left(D_{x}^{2}+\bar{D}_{x}^{2}\right) \delta^{4}\left(\theta_{x}-\theta_{y}\right)=0,
$$

из которого следует, что

$$
P_{2}(p)=F_{2}(p)-\frac{1}{2 e^{2} \xi} p^{2},
$$

тогда как функция $P_{1}$, соответствующая калибровочно-инвариантному контрчлену, не может быть определена из тождества Уорда. Удобно выбрать

$$
P_{1}(p)=F_{1}\left(\mu_{\pi}\right)-\frac{1}{2 e^{2} \xi} p^{2},
$$

где $\mu_{\pi}$ есть точка нормировки, которая, вообще говоря, может отличаться от $\mu_{\sigma}$. В этом случае перенормированная двухточечная функция Грина может быть записана как

$$
\begin{aligned}
\Pi^{r}\left[\left(\theta_{x}, p\right),\left(\theta_{y},-p\right)\right]= & \frac{1}{32 e^{2} \xi}\left(D^{2} \bar{D}^{2}+\bar{D}^{2} D^{2}\right) \delta^{4}\left(\theta_{x}-\theta_{y}\right)+ \\
& +\left(F_{1}(p)-F_{1}\left(\mu_{\pi}\right)\right) p^{2} \Pi_{1 / 2} \delta^{4}\left(\theta_{x}-\theta_{y}\right)
\end{aligned}
$$

Такая функция Грина удовлетворяет уравнению

$$
\left(D_{x}^{2}+\bar{D}_{x}^{2}\right)\left(\Pi^{r}\left[\left(\theta_{x}, p\right),\left(\theta_{y},-p\right)\right]-\frac{1}{2 e^{2} \xi} p^{2} \delta^{4}\left(\theta_{x}-\theta_{y}\right)\right)=0
$$

которое является суперсимметричным обобшением условия поперечности поляризационного оператора в обычной квантовой электродинамике.

Функции Грина, содержашие только внешние $V$-линии с $E_{V}>2$, перенормируются аналогичным образом. Для этого достаточно положить в уравнениях $(25)$ и $(26) R_{j}=0$. В этом случае соотношение (22) можно использовать как определение перенормированной функции $\Pi^{r}$.

Таким образом, построение процедуры перенормировки завершено. В силу локальности выгитаемых членов в пределе, когда снимается регуляризация, предлагаемая схема эквивалентна добавлению контрчленов

$$
\begin{aligned}
\Delta S= & -\sum_{n, i} \frac{1}{n !} \int d^{4} x d^{4} \theta_{1} \ldots d^{4} \theta_{n} B_{i}(\theta, \partial) P_{i}(\partial) V_{1}\left(\theta_{1}, x\right) \ldots V\left(\theta_{n}, x\right)+ \\
& +\frac{1}{4} \sum_{n=0}^{\infty}\left(Z_{\Gamma_{n}}-1\right) \frac{1}{n !} \int d^{4} x d^{4} \theta \phi^{*}(2 V)^{n} \phi+ \\
& +\frac{1}{4} \sum_{n=0}^{\infty}\left(\widetilde{Z}_{\Gamma_{n}}-1\right) \frac{1}{n !} \int d^{4} x d^{4} \theta \tilde{\phi}^{*}(-2 V)^{n} \tilde{\phi}
\end{aligned}
$$

4 Теоретическая и математическая физика, т. 135, № 2, 2003 г. 
Важно заметить, что благодаря присутствию $\delta$-функций в $B_{i}$ все слагаемые в этом выражении могут быть представлены в виде интегралов по единственному $\theta$. При этом в случае использования неивариантной регуляризации эти контрчлены, конечно, могут оказаться не калибровочно-инвариантными.

После построения однопетлевых контрчленов можно вычислить двухпетлевые диаграммы и провести аналогичную перенормировку на двухпетлевом уровне. При этом вся комбинаторика определяется стандартной $R$-операшией. После перенормировки в каждой петле перенормированные функции Грина будут автоматически удовлетворять перенормированным тождествам Уорда. Это означает, что предлагаемая схема будет давать калибровочно-инвариантный результат даже при использовании регуляризации, которая нарушает калибровочную инвариантность.

\section{4. ПРИМЕНЕНИЕ УНИВЕРСАЛЬНОЙ КАЛИБРОВОЧНО-ИНВАРИАНТНОЙ СХЕМЫ ПЕРЕНОРМИРОВКИ НА ОДНОПЕТЛЕВОМ УРОВНЕ}

Для того чтобы проиллюстрировать применение описанной вьше схемы перенормировки, рассмотрим однопетлевую перенормировку $N=1$ суперсимметричной квантовой электродинамики, регуляризованной при помоши обрезания петлевого импульса в калибровке $\xi=-1$.

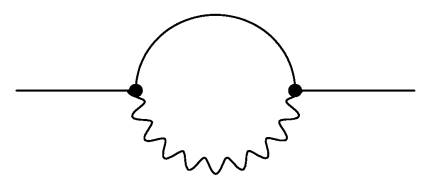

Рис. 1. Диаграмма Фейнмана, дающая нетривиальный вклад в однопетлевую аномальную размерность.

Диаграмма, определяюшая однопетлевую двухточечную функцию Грина суперполя материи представлена на рис. 1. После несложных вычислений с использованием правил Фейнмана в суперпространстве, описанных в книге [20], в евклидовом пространстве вклад этой диаграммы в эффективное действие может быть записан в виде

$$
\begin{aligned}
\Delta \Gamma_{\phi}^{(1)}= & -\int \frac{d^{4} q}{(2 \pi)^{4}} d^{4} \theta e^{2}\left(\phi^{*}(q) \phi(-q)+\tilde{\phi}^{*}(q) \phi(-q)\right) \times \\
& \times \int^{M} \frac{d^{4} k}{(2 \pi)^{4}} \frac{1}{2(k+q)^{2}\left(k^{2}+m^{2}\right)},
\end{aligned}
$$

где интегрирование по петлевому импульсу $k$ определяется как

$$
\int^{M} d^{4} k \equiv \int_{0}^{M} d k k^{3} \int_{0}^{\pi} d \theta_{1} \sin ^{2} \theta_{1} \int_{0}^{\pi} d \theta_{2} \sin \theta_{2} \int_{0}^{2 \pi} d \theta_{3}
$$


а $M$ является параметром ультрафиолетового обрезания. Поэтому в соответствии с (18) в однопетлевом приближении функция $\Sigma(q)$ оказывается равной

$$
\begin{aligned}
\Sigma(q) & =-\int^{M} \frac{d^{4} k}{(2 \pi)^{4}} \frac{e^{2}}{8(k+q)^{2}\left(k^{2}+m^{2}\right)}= \\
& =-\frac{e^{2}}{128 \pi^{2}}\left(\ln \frac{M^{2}+m^{2}}{q^{2}+m^{2}}+1-\frac{m^{2}}{q^{2}} \ln \frac{q^{2}+m^{2}}{m^{2}}\right) .
\end{aligned}
$$

Двухточечная функция суперполя материи перенормируется при помоши вычитания (20), которое соответствует

$$
\Delta S=\frac{\alpha}{8 \pi}\left(\ln \frac{M^{2}+m^{2}}{\mu_{\sigma}^{2}+m^{2}}+1-\frac{m^{2}}{\mu_{\sigma}^{2}} \ln \frac{\mu_{\sigma}^{2}+m^{2}}{m^{2}}\right) \int d^{4} x d^{4} \theta\left(\phi^{*} \phi+\tilde{\phi}^{*} \tilde{\phi}\right)
$$

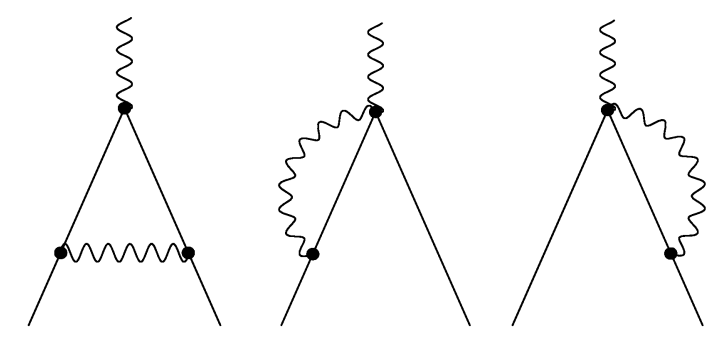

Рис. 2. Диаграммы Фейнмана, дающие нетривиальный вклад в однопетлевую трехточечную вершинную функцию в $N=1$ суперсимметричной электродинамике.

Затем необходимо провести перенормировку однопетлевой трехточечной функции, которая определяется диаграммами, показанными на рис. 2. После их вычисления при использовании регуляризации путем обрезания петлевого импульса было получено, что соответствуюшая трехточечная функция Грина может быть записана как

$$
\begin{aligned}
\Gamma\left[\left(\theta_{x}, p\right) ;\left(\theta_{y}, q\right),\left(\theta_{z},-q-p\right)\right]= \\
=\left\{-\int^{M} \frac{d^{4} k}{(2 \pi)^{4}}\left(\frac{e^{2}}{8 k^{2}\left((k+q)^{2}+m^{2}\right)}+\frac{e^{2}}{8 k^{2}\left((k+q+p)^{2}+m^{2}\right)}\right)-\right. \\
\quad-\int^{M} \frac{d^{4} k}{(2 \pi)^{4}} \frac{e^{2}(k+q+p / 2)_{\mu}}{4 k^{2}\left((k+q)^{2}+m^{2}\right)\left((k+q+p)^{2}+m^{2}\right)} \frac{1}{4} \bar{D}_{x} \gamma^{\mu} \gamma_{5} D_{x}+ \\
\left.\quad+\int^{M} \frac{d^{4} k}{(2 \pi)^{4}} \frac{e^{2}}{8 k^{2}\left((k+q)^{2}+m^{2}\right)\left((k+q+p)^{2}+m^{2}\right)} p^{2}\left(\Pi_{1 / 2}\right)_{x}\right\} \times \\
\quad \times\left(\bar{D}_{x}^{2} \delta^{4}\left(\theta_{y}-\theta_{x}\right) D_{x}^{2} \delta^{4}\left(\theta_{x}-\theta_{z}\right)\right) .
\end{aligned}
$$


При этом структурные функции $B_{i}$, входящие в соотношение $(21)$, могут быть выбраны в виде

$$
\begin{aligned}
& B_{1}(\theta, p, q)=\bar{D}_{x}^{2} \delta^{4}\left(\theta_{y}-\theta_{x}\right) D_{x}^{2} \delta^{4}\left(\theta_{x}-\theta_{z}\right), \\
& B_{2}(\theta, p, q)=\frac{1}{4} \bar{D}_{x} \gamma^{\mu} \gamma_{5} D_{x}\left(\bar{D}_{x}^{2} \delta^{4}\left(\theta_{y}-\theta_{x}\right) D_{x}^{2} \delta^{4}\left(\theta_{x}-\theta_{z}\right)\right), \\
& B_{3}(\theta, p, q)=p^{2}\left(\Pi_{1 / 2}\right)_{x}\left(\bar{D}_{x}^{2} \delta^{4}\left(\theta_{y}-\theta_{x}\right) D_{x}^{2} \delta^{4}\left(\theta_{x}-\theta_{z}\right)\right) .
\end{aligned}
$$

Перенормированная вершинная функция получается после вычитания соответствующих полиномов:

$$
\begin{aligned}
\Gamma_{3}^{r}\left[\left(\theta_{x}, p\right) ;\left(\theta_{y}, q\right),\left(\theta_{z},-q-p\right)\right]= \\
=\left\{-\int^{M} \frac{d^{4} k}{(2 \pi)^{4}}\left(\frac{e^{2}}{8 k^{2}\left((k+q)^{2}+m^{2}\right)}+\frac{e^{2}}{8 k^{2}\left((k+q+p)^{2}+m^{2}\right)}\right)-P_{1}(p)+\right. \\
\quad+\left(-\int^{M} \frac{d^{4} k}{(2 \pi)^{4}} \frac{1}{4 k^{2}\left((k+q)^{2}+m^{2}\right)\left((k+q+p)^{2}+m^{2}\right)}\right) \frac{1}{4} \bar{D}_{x} \gamma^{\mu} \gamma_{5} D_{x}+ \\
\left.\quad+\left(\int^{M} \frac{d^{4} k}{(2 \pi)^{4}} \frac{e^{2}}{8 k^{2}\left((k+q)^{2}+m^{2}\right)\left((k+q+p)^{2}+m^{2}\right)}\right) p^{2}\left(\Pi_{1 / 2}\right)_{x}\right\} \times \\
\quad \times\left(\bar{D}_{x}^{2} \delta^{4}\left(\theta_{y}-\theta_{x}\right) D_{x}^{2} \delta^{4}\left(\theta_{x}-\theta_{z}\right)\right) .
\end{aligned}
$$

С учетом соотношений

$$
\begin{gathered}
D^{2} \bar{D} \gamma_{\mu} \gamma_{5} D=4 i D^{2} \partial_{\mu}, \\
\bar{D}^{2} \bar{D} \gamma_{\mu} \gamma_{5} D=-4 i \bar{D}^{2} \partial_{\mu}, \\
\bar{D}^{2} \Pi_{1 / 2}=D^{2} \Pi_{1 / 2}=0
\end{gathered}
$$

левая часть тождества Уорда оказывается равной

$$
\begin{aligned}
\left(D_{x}^{2}+\right. & \left.\bar{D}_{x}^{2}\right) \Gamma_{3}^{r}\left[\left(\theta_{x}, p\right) ;\left(\theta_{y}, q\right),\left(\theta_{z},-q-p\right)\right]= \\
= & \bar{D}_{x}^{2} \delta^{4}\left(\theta_{y}-\theta_{x}\right) \bar{D}_{x}^{2} D_{x}^{2} \delta^{4}\left(\theta_{x}-\theta_{z}\right) \times \\
& \times\left(-\int^{M} \frac{d^{4} k}{(2 \pi)^{4}} \frac{e^{2}}{8 k^{2}\left((k+q+p)^{2}+m^{2}\right)}-P_{1}(p)\right)+ \\
& +D_{x}^{2} \delta^{4}\left(\theta_{x}-\theta_{z}\right) D_{x}^{2} \bar{D}_{x}^{2} \delta^{4}\left(\theta_{x}-\theta_{y}\right) \times \\
& \times\left(-\int^{M} \frac{d^{4} k}{(2 \pi)^{4}} \frac{e^{2}}{8 k^{2}\left((k+q)^{2}+m^{2}\right)}-P_{1}(p)\right)
\end{aligned}
$$

тогда как правая часть тождества Уорда записывается в виде

$$
2 \bar{D}_{x}^{2} \delta^{4}\left(\theta_{y}-\theta_{x}\right) \bar{D}_{x}^{2} D_{x}^{2} \delta^{4}\left(\theta_{x}-\theta_{z}\right) \Sigma^{r}(q+p)+2 D_{x}^{2} \delta^{4}\left(\theta_{x}-\theta_{z}\right) D_{x}^{2} \bar{D}_{x}^{2} \delta^{4}\left(\theta_{x}-\theta_{y}\right) \Sigma^{r}(q) .
$$

Таким образом, в этом случае

$$
\begin{aligned}
& Q_{1}(\theta, p, q)=\bar{D}_{x}^{2} \delta^{4}\left(\theta_{y}-\theta_{x}\right) \bar{D}_{x}^{2} D_{x}^{2} \delta^{4}\left(\theta_{x}-\theta_{z}\right), \\
& Q_{2}(\theta, p, q)=D_{x}^{2} \delta^{4}\left(\theta_{x}-\theta_{z}\right) D_{x}^{2} \bar{D}_{x}^{2} \delta^{4}\left(\theta_{x}-\theta_{y}\right) .
\end{aligned}
$$


Принимая во внимание, что

$$
\Sigma^{r}(q)=-\int^{M} \frac{d^{4} k}{(2 \pi)^{4}} \frac{e^{2}}{8 k^{2}\left((k+q)^{2}+m^{2}\right)}-\Sigma\left(\mu_{\sigma}\right)
$$

получаем

$$
P_{1}(p)=\Sigma\left(\mu_{\sigma}\right)
$$

Поэтому перенормированная трехточечная функция Грина оказывается равной

$$
\begin{aligned}
\Gamma^{r} & {\left[\left(\theta_{x}, p\right) ;\left(\theta_{y}, q\right),\left(\theta_{z},-q-p\right)\right]=\left\{\Sigma^{r}(q)+\Sigma^{r}(q+p)-\right.} \\
& -\int \frac{d^{4} k}{(2 \pi)^{4}} \frac{e^{2}(k+q+p / 2)_{\mu}}{4 k^{2}\left((k+q)^{2}+m^{2}\right)\left((k+q+p)^{2}+m^{2}\right)} \frac{1}{4} \bar{D}_{x} \gamma^{\mu} \gamma_{5} D_{x}+ \\
& \left.+\int \frac{d^{4} k}{(2 \pi)^{4}} \frac{e^{2}}{8 k^{2}\left((k+q)^{2}+m^{2}\right)\left((k+q+p)^{2}+m^{2}\right)} p^{2}\left(\Pi_{1 / 2}\right)_{x}\right\} \times \\
& \times\left(\bar{D}_{x}^{2} \delta^{4}\left(\theta_{y}-\theta_{x}\right) D_{x}^{2} \delta^{4}\left(\theta_{x}-\theta_{z}\right)\right) .
\end{aligned}
$$

Очевидно, что проведенное вычитание (вместе с соответствующим вычитанием для $\widetilde{\Gamma})$ эквивалентно добавлению следующих контрчленов:

$$
\Delta S=\frac{\alpha}{8 \pi}\left(\ln \frac{M^{2}+m^{2}}{\mu_{\sigma}^{2}+m^{2}}+1-\frac{m^{2}}{\mu_{\sigma}^{2}} \ln \frac{\mu_{\sigma}^{2}+m^{2}}{m^{2}}\right) \int d^{4} x d^{4} \theta\left(\phi^{*} 2 V \phi-\tilde{\phi}^{*} 2 V \tilde{\phi}\right)
$$

где было использовано явное выражение для $\Sigma\left(\mu_{\sigma}\right)$, построенное ранее. Тем самым завершено построение перенормированной трехточечной функции Грина. На следующем шаге необходимо рассмотреть четырехточечную функцию с двумя внешними линиями суперполей материи и продолжить рекуррентный процесс.

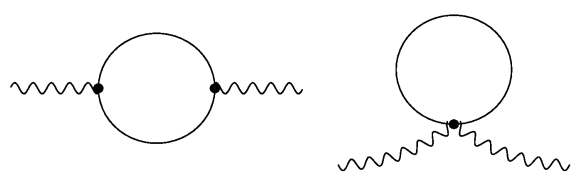

Рис. 3. Диаграммы Фейнмана, дающие нетривиальный вклад в однопетлевую $\beta$-функцию $N=1$ суперсимметричной электродинамики.

Однопетлевой вклад в двухточечную функцию калибровочного поля определяется диаграммами, приведенными на рис. 3 , и оказывается равным

$$
\begin{aligned}
& \int \frac{d^{4} p}{(2 \pi)^{4}}\left\{\int d^{2} \theta W_{a}(p) C_{a b} W_{b}(-p) \int^{M} \frac{d^{4} k}{(2 \pi)^{4}} \frac{1}{2\left(k^{2}+m^{2}\right)\left((k+p)^{2}+m^{2}\right)}+\right. \\
& \left.+\int d^{4} \theta V(p) V(-p) \int^{M} \frac{d^{4} k}{(2 \pi)^{4}}\left(\frac{1}{(k+p)^{2}+m^{2}}-\frac{1}{k^{2}+m^{2}}\right)\right\}
\end{aligned}
$$


Заметим, что благодаря использованию регуляризации, которая нарушает калибровочную инвариантность, в формуле (55) присутствуют неинвариантные слагаемые. Выражение (55) соответствует

$$
\begin{gathered}
F_{1}(p)=\int^{M} \frac{d^{4} k}{(2 \pi)^{4}} \frac{1}{\left(k^{2}+m^{2}\right)\left((k+p)^{2}+m^{2}\right)}, \\
F_{2}(p)=\int^{M} \frac{d^{4} k}{(2 \pi)^{4}}\left(\frac{2}{(k+p)^{2}+m^{2}}-\frac{2}{k^{2}+m^{2}}\right)=-\frac{p^{2}}{16 \pi^{2}}+O\left(\frac{1}{M^{2}}\right)
\end{gathered}
$$

в формуле (30). (Получить явное выражение для функции $F_{1}(p)$ достаточно несложно, однако оно является громоздким и здесь не приводится.) Перенормированный вклад диаграмм с двумя внешними $V$-линиями в эффективное действие (37) может быть представлен в виде

$$
\frac{1}{2} \int \frac{d^{4} p}{(2 \pi)^{4}} d^{2} \theta W_{a}(p) C_{a b} W_{b}(-p)\left(F_{1}(p)-F_{1}\left(\mu_{\pi}\right)\right),
$$

причем все неинвариантные члены исчезают и результат оказывается конечным. При этом $\beta$-функция, соответствуюшая формуле (57), равна

$$
\beta=\frac{\alpha^{2}}{\pi}+O\left(\alpha^{3}\right)
$$

и согласуется с вычислениями, выполненными в рамках размерной редукции [20].

Несложно проверить, что проведенное вычитание эквивалентно добавлению контрчленов

$$
\Delta S=-\frac{1}{2} F_{1}\left(\mu_{\pi}\right) \operatorname{Re} \int d^{4} x d^{2} \theta W_{a} C_{a b} W_{b}-\frac{1}{32 \pi^{2}} \int d^{4} x d^{4} \theta V \partial^{2} V,
$$

которые не являются калибровочно-инвариантными.

\section{5. ЗАКЛЮЧЕНИЕ}

В данной работе была предложена схема перенормировки для суперсимметричной квантовой электродинамики, которая гарантирует калибровочную инвариантность перенормированной теории для произвольной промежуточной регуляризации. При этом суперсимметричные тождества Уорда включаются в вычитания, которые позволяют избежать появления неинвариантных контрчленов.

Такая же процедура может быть применена к неабелевым суперсимметричным теориям, сформулированным в терминах соответствуюших суперполей. Технические проблемы, связанные с более сложной структурой неабелевых теорий, будут рассмотрены отдельно.

Благодарности. Работа частично поддержана грантом РФФИ № 02-01-00126 и Президентским грантом поддержки ведуших научных школ № НШ 2052.2003.1. 


\section{Список литературы}

[1] А. А. Славнов. ТМФ. 1975. Т. 23. № 1. С. 3.

[2] A. A. Slavnov. Nucl. Phys. B. 1975. V. 97. P. 155.

[3] S. Ferrara, O. Piguet. Nucl. Phys. B. 1975. V. 93. P. 261.

[4] В.К. Кривощеков, А. А. Славнов, Б. А. Файзуллаев. ТМФ. 1976. Т. 26. № 2. С. 147.

[5] G. 't Hooft, M. Veltman. Nucl. Phys. B. 1972. V. 44. P. 189.

[6] W. Siegel. Phys. Lett. B. 1979. V. 84. P. 193.

[7] I. Jack, D. Jones. Regularization of supersymmetric theories. In: Perspectives of Supersymmetry. Ed. G.L. Kane. Singapore: World Scientific, 1998. P. 149; hep-ph/9707278.

[8] A. Slavnov. Nucl. Phys. B. 1971. V. 31. Р. 301; A. A. Славнов. ТМФ. 1972. Т. 13. № 2. C. 174 .

[9] T. Bakeyev, A. Slavnov. Mod. Phys. Lett. A. 1996. V. 11. P. 1539.

[10] P. West. Nucl. Phys. B. 1986. V. 268. P. 113.

[11] А. А. Солошенко, К. В. Степаньяни. ТМФ. 2002. Т. 131. № 1. С. 135.

[12] A. Soloshenko, K. Stepanyantz. Two-loop renormalization of $N=1$ supersymmetric electrodynamics, regularized by higher derivatives. hep-th/0203118.

[13] D. Z. Freedman, K. Johnson, J. I. Latorre. Nucl. Phys. B. 1992. V. 371. P. 353.

[14] J. Mas, M. Perez-Victoria, C. Seijas. JHEP. 2002. V. 0203. P. 049; hep-th/0202082.

[15] O. Piguet, K. Sibold. Nucl. Phys. B. 1985. V. 253. P. 517.

[16] O. Piguet, K. Sibold. Nucl. Phys. B. 1984. V. 248. P. 301.

[17] N. Maggiore. Int. J. Mod. Phys. A. 1995. V. 10. P. 3781.

[18] A. A. Slavnov. Phys. Lett. B. 2001. V. 518. P. 195.

[19] А. А. Славнов. ТМФ. 2002. Т. 130. № 1. С. 3.

[20] П. Уэст. Введение в суперсимметрию и супергравитацию. М.: Мир, 1989.

[21] T. E. Clark, O. Piquet, K. Sibold. Nucl. Phys. B. 1980. V. 169. P. 77; V. 174. P. 491.

Поступила в редакцию 1.VIII.2002 г. 